\title{
Protective Effect of a Bispecific Fc-Fusion Protein on the Barrier of Human Retinal Pigment Epithelial Cells
}

\author{
Jing Wang ${ }^{a}$ Yishen Wang ${ }^{a}$ Dechao Yu ${ }^{b}$ Qiuhui Liu ${ }^{a}$ Shaofen Lin ${ }^{a}$ \\ Rong Tian ${ }^{\mathrm{a}} \mathrm{Jia} \mathrm{Li}^{\mathrm{b}}$ Yan Luo ${ }^{\mathrm{a}}$ \\ aState Key Laboratory of Ophthalmology, Zhongshan Ophthalmic Center, Sun Yat-sen University, Guangzhou, \\ China; bState Key Laboratory of Biotherapy, West China Hospital, Sichuan University, Chengdu, China
}

\section{Keywords}

hRPE · IBI302 - Vascular endothelial growth factor

\begin{abstract}
Introduction: The aim of the study was to evaluate the protective effects of IBI302, a bispecific Fc-fusion protein that theoretically can bind vascular endothelial growth factor (VEGF), complement C3b, and C4b in the barrier of the cultured human retinal pigment epithelial (hRPE) cells. Methods: Primary hRPE cells were isolated and cultured to monolayer barrier. hRPE monolayers were divided into the PBS control group, VEGF-Trap group, complement receptor 1 (CR1) group, and IBI302 group. Identification of hRPE cells, barrier function, inflammation factors, and immune response products was tested by immunofluorescent staining, transepithelial resistance (TER), and ELISA. Results: IBI302 treatment significantly improved the TER of the barrier of hRPE cells after complement-activated oxidative stress compared with the PBS control group, VEGF-Trap group, and CR1 group. The maximum effect of IBI302 on protecting hRPE cell viability was observed at the concentration of $1 \mu \mathrm{g} / \mathrm{mL}$. The elevated expression of VEGF, chemokine (C-C Motif) ligand $2, \mathrm{C} 3 \mathrm{a}, \mathrm{C} 5 \mathrm{a}$, and membrane attack complex was reduced by
\end{abstract}

IBI302. Conclusion: IBI302 could protect the barrier function of hRPE cells. IBI302 might be a potentially effective drug for the RPE barrier-associated ocular diseases.

C 2021 S. Karger AG, Basel

\section{Introduction}

Age-related macular degeneration (AMD), the main cause of irreversible blindness in individuals over the age of 55 years, is characterized by the progressive degeneration of retinal pigment epithelial (RPE). Aging, family history, cigarette smoking, sunlight exposure, increased oxidative stress, and complement risk alleles [1-3] are thought to impact RPE function [4]. In addition, the elevated levels of vascular endothelial growth factor (VEGF), inflammation cytokines such as tumor necrosis factor alpha and chemokine (C-C Motif) ligand 2 (CCL2), and the local leukocyte infiltration are also considered playing important roles in the pathogenesis of RPE atrophy $[5,6]$.

The anti-oxidative capacity of RPE cells will decline over the years due to the accumulation of oxidative damage caused by the constant exposure to high energetic

Correspondence to:

Yan Luo, luoyan2@mail.sysu.edu.cn 
sunlight and its high metabolic activity and the associated oxygen consumption [7]. Recent studies have identified the critical role of the complement cascade in oxidative stress and the inflammatory response in AMD [8]. Complement (C) components, such as $\mathrm{C} 3, \mathrm{C} 5$, and the membrane attack complex (MAC), have been found in drusen as hallmark of dry AMD [9] and in the mammalian serum $[10,11]$. Oxidative stress, VEGF, and complement act synergistically to generate an aggressive inflammatory environment at the level of the RPE-Bruch's membrane interface [12]. C3a and C5a, the bioactive fragments of C3 and C5, can upregulate the secretion of VEGF in RPE cells [13]. MAC is reported to promote the release of growth factors such as $\beta$-fibroblast growth factor and VEGF [14]. Interestingly, the RPE cells become more susceptible to complement-mediated damage [15].

IBI302 (Innovent Biologics Inc.; Suzhou, China) is a novel bispecific decoy receptor fusion protein designed with a VEGF inhibition domain and a complement cascade inhibition domain fusing to the Fc portion of human immunoglobulin. The VEGF inhibition domain combines the extracellular domain 2 of VEGFR1 and the extracellular domain 3 of VEGFR2. The complement cascade inhibition domain comprises $3 \mathrm{C} 3 \mathrm{~b}$ and $\mathrm{C} 4 \mathrm{~b}$ binding sites of complement receptor 1 (CR1). CR1 acts as a cofactor to mediate the cleavage of both $\mathrm{C} 4 \mathrm{~b}$ and $\mathrm{C} 3 \mathrm{~b}$. It also has decay-accelerating activity against $\mathrm{C} 3$ convertases [16]. A previous study has already confirmed that IBI302 can bind different VEGF isoforms including VEGF165, VEGF121, and PIGF and complement proteins C3b and C4b with high affinity [17]. IBI302 has showed an inhibitory effect on the VEGF165-induced cell proliferation of human primary umbilical vein endothelia and the activation of classical complement pathway and the alternative complement pathway in vitro [17]. Based on the critical role of complement in the inflammation of RPE cells, this study aimed to investigate the protective effect and its underlying mechanisms of IBI302 on human retinal pigment epithelial (hRPE) monolayer under the complement-activated oxidative stress.

\section{Materials and Methods}

In vitro hRPE Monolayer Barrier Model under the Complement Activated-Oxidative Stress

RPE cells were isolated from donors' eyes from Eye Bank of Zhongshan Ophthalmic Center as described previously [18], then resuspended in human DMEM/F12 (Gibco, Grand Island, NY, USA) with $10 \%$ fetal bovine serum (FBS), and then seeded on a transwell insert at $37^{\circ} \mathrm{C}$ in a $5 \% \mathrm{CO}_{2}$ incubator. The medium was changed every 2 days. All cell experiments were carried out using the passage 3-5 hRPE.

The hRPE cells were cultured on 12-well transwell $(12 \mathrm{~mm}$, pore size $0.4 \mu \mathrm{m}$; Corning, NY, USA) insert with DMEM/F12 containing $10 \%$ FBS, then changed to DMEM/F12 containing $1 \%$ FBS when they became confluent, and then cultured for about additional 2 weeks to establish a stable monolayer. The cell monolayer was considered stable when the transepithelial resistance (TER) was repeatedly measured as $55-60 \Omega \mathrm{cm}^{2}$ [19]. To eliminate the effect of FBS on complement activation, the stable hRPE monolayer was cultured in serum-deprived DMEM/F12 for another 1 week as described previously [19]. Cells on the transwell insert were fixed in $4 \%$ paraformaldehyde for immunofluorescence staining. Mouse antihuman RPE65 (Novus, Littleton, CO, USA) antibody was used to identify hRPE by immunofluorescence staining. The hRPE monolayer barrier was exposed to $500 \mu \mathrm{M}$ ten-butyl hydroperoxide (t-BHP) and 10\% normal human serum (NHS) for $4 \mathrm{~h}$ to establish the in vitro hRPE monolayer barrier model under complement-activated oxidative stress according to some published studies [20,21]. The untreated hRPE monolayer was used as a normal blank control.

\section{MTT Assay for hRPE Cell Viability under Different} Concentrations of IBI302

The 3-[4,5-dimethylthiazol-2-yl]-2,5-diphenyltetrazolium bromide (MTT) assay was used to measure hRPE cells' viability under complement-activated oxidative stress to find the optimal concentration of IBI302. hRPE cells were seeded on 96-well plates $\left(1 \times 10^{4}\right.$ cells/well $)$. After the hRPE cells reach about $80 \%$ confluence, $500 \mu \mathrm{M}$ t-BHP and $10 \%$ NHS were added to mimic complement-activated oxidative stress in vitro. The hRPE cells were immediately treated with the different concentrations of IBI302 (10 $\mathrm{ng} / \mathrm{mL}, 100 \mathrm{ng} / \mathrm{mL}, 500 \mathrm{ng} / \mathrm{mL}, 1 \mu \mathrm{g} / \mathrm{mL}$, and $10 \mu \mathrm{g} / \mathrm{mL}$; Innovent Biologics, Inc. Suzhou, China) or PBS for $4 \mathrm{~h}$. A normal cultured hRPE monolayer with PBS treatment but without the treatment of $\mathrm{t}$-BHP and NHS was used as a normal blank control. The media were carefully aspirated, and then $200 \mu \mathrm{L}$ of $0.5 \mathrm{mg} / \mathrm{mL}$ MTT (Sigma-Aldrich, St. Louis, MO, USA) was added into the wells and incubated for $4 \mathrm{~h}$, followed by the additional $150 \mu \mathrm{L}$ of dimethyl sulphoxide (Sigma-Aldrich, St. Louis, MO, USA). The absorbance at $550 \mathrm{~nm}$ was determined by a microtiter plate reader (Elx800, BioTEK Ins, North Brunswick, NJ, USA). Well without cells was used as a blank contrast. All data were subtracted by the OD of blank well before the calculation. The percentage of cell viability was calculated as (OD of treated sample/OD of normal blank group) $\times 100$.

IBI302 Treatment for hRPE Monolayer under the Complement Activated-Oxidative Stress

The hRPE monolayer cells were divided into 4 groups: PBS control group, IBI302-treated group, VEGF-Trap-treated group, and CR1-treated group. According to previously MTT assay, $1 \mu \mathrm{g} /$ $\mathrm{mL}$ of IBI302, $1 \mu \mathrm{g} / \mathrm{mL}$ of VEGF-Trap (Eylea ${ }^{\mathrm{TM}}$, Regeneron pharmaceuticals, Inc., Tarrytown, NY, USA), and $1 \mu \mathrm{g} / \mathrm{mL}$ of CR1 (Innovent Biologics, Inc., Suzhou, China) were immediately added to the apical chambers of transwells after t-BHP and NHS were added, respectively, and incubated for $4 \mathrm{~h}$. The same volume of PBS was added to the PBS control group.

TER Measurement for the Barrier Function of hRPE Monolayer

The hRPE cells were cultured as a mature monolayer on 12well transwell inserts. Since TER is a function indicator of the bar- 
rier of RPE cells and complement activation results in the dysfunction of barrier [22], TER was measured to estimate the effect of IBI302 on the barrier function of the hRPE monolayer under complement activated-oxidative stress. The TER of the monolayer cell on the transwell insert was determined by measuring the resistance across the monolayer with an EVOM volt-ohmmeter (World Precision Instruments, Sarasota, FL, USA). The TER value of cell monolayer was determined by subtracting the TER of inserts without cells and then multiplying by the surface area of the inserts [19].

ELISA for the Levels of VEGF, Inflammation Factors, and Immune Response Products

For the ELISA assay, cell culture supernatant in the apical chamber of transwells in each group was collected $4 \mathrm{~h}$ after treatment and then added the protease inhibitor (Roche, Basel, Switzerland) and FUT-175 (BD Biosciences, Franklin, NJ, USA). The levels of C3a, C5a, MAC, VEGF, and CCL2 were determined by the human C3a, C5a, MAC ELISA kits (Quidel, San Diego, CA, USA), and human VEGF and CCL2 ELISA kits (R\&D Systems Inc., Thermo, Minneapolis, MN, USA). Data were normalized by the total protein which was determined by Micro BCA protein assay kit (Micro BCA, Thermo, Waltham, MA, USA).

\section{Statistical Analysis}

All values are expressed as mean \pm standard deviation. Oneway ANOVA was used for multiple comparisons. For those experiments with only 2 individual groups, $t$ test was performed to determine the significance. For those experiments with $>2$ individual groups, least statistical difference test was performed for comparison between 2 or more pairs of means. A value of $p<0.05$ was considered statistically significant. The experiments were repeated 3 times with 3 different batches of objects.

\section{Results}

\section{The Identification of $h R P E$ and Establishment of}

\section{$h R P E$ Monolayer Barrier}

On the second day after inoculation, primary cultured hRPE cells at passage 4 were polygonal with some pigment particles in partial cells shown in an inverted phase contrast microscope (shown in Fig. 1a, A1). Under confocal fluorescence microscope, most of the cultured cells were labeled by RPE65 (a specific marker of RPE, red fluorescence) with DAPI-stained nuclear (blue fluorescence) (shown in Fig. 1a, A2). Cultured on the transwell inserts about 21 days, hRPE cells were closely packed hexagonal and formed a monolayer barrier, with pigment particles in cytoplasm (shown in Fig. 1a, A3, A4).

Oxidative Stress in Combination with NHS Disrupted the Barrier Function of hRPE Cells

It is reported that oxidative stress renders RPE cells susceptible to complement-mediated injury [23]. To de- termine at which time point does oxidative stress in combination with NHS acquire the most effect on disruption of the hRPE monolayer barrier, TER was recorded from 0 to $6 \mathrm{~h}$ after $500 \mu \mathrm{M} \mathrm{t}$-BHP and 10\% NHS treatment (shown in Fig. 1b). The untreated hRPE cells were used as control. No significant difference existed among the TER at all the time points in the untreated hRPE cells. While the TER of the untreated hPRE cells at 1,2, 4, and $6 \mathrm{~h}$ were significantly higher than that of the t-BHP and NHS-treated hRPE cells. Oxidative stress in combination with NHS reduced TER in a time-dependent manner, with the lowest value at $4 \mathrm{~h}$ and a little rebound at $6 \mathrm{~h}$. TER at $0 \mathrm{~h}, 1 \mathrm{~h}$, and $2 \mathrm{~h}$ was significantly higher than that at 4 h (both $p<0.005$ ); however, no significant difference existed between the TER at 4 and $6 \mathrm{~h}(p=0.252)$. Therefore, 4-h exposure to the complement-activated oxidative stress was chosen as the time point for the following experiments.

\section{One Microgram per Milliliter of IBI302 Maintained the Cell Viability of hRPE under the Complement- \\ Activated Oxidative Stress}

To determine the optimal concentration of IBI302 in vitro model, cell viability was measured by MTT assay (shown in Fig. 1c). Compared to the normal blank group, the cell viability of RPE cells in t-BHP + NHS group (500 $\mu \mathrm{M} \mathrm{t}-\mathrm{BHP}+10 \% \mathrm{NHS})$ showed a significant decrease to $52.35 \pm 1.30 \%$ ( $p<0.001$ vs. normal). Compared with that of the normal blank group, the cell viability of RPE cells in $10 \mathrm{ng} / \mathrm{mL}, 100 \mathrm{ng} / \mathrm{mL}, 500 \mathrm{ng} / \mathrm{mL}, 1 \mu \mathrm{g} / \mathrm{mL}$, and $10 \mu \mathrm{g} /$ $\mathrm{mL}$ of the IBI302-treated t-BHP + NHS group increased to $55.54 \pm 2.78 \%, 61.37 \pm 4.57 \%, 64.93 \pm 4.42 \%, 73.87 \pm$ $4.13 \%$, and $70.38 \pm 3.74 \%$, respectively ( $p<0.05$ vs. normal blank group). The IBI302 treated groups with different concentrations had a slight rise in the cell viability. No significant difference existed between 1 and $10 \mu \mathrm{g} / \mathrm{mL}$ group. Therefore, $1 \mu \mathrm{g} / \mathrm{mL}$ of IBI302 was chosen for the further experiments, and the same concentration of VEGF-Trap and CR1 was used as controls.

IBI302 Restored the TER of RPE Monolayer under the Complement Activated-Oxidative Stress

To determine the effect of IBI302 on the hRPE monolayer, TER was measured $4 \mathrm{~h}$ after IBI302 treatment (shown in Fig. 1d). The average value of TER for the normal blank control, PBS control, VEGF-Trap, CR1, and IBI302 group was $59,31,38,37$, and $44 \mathrm{~cm}^{2}$, respectively. Consistent with previous studies [24], the PBS control group reduced TER by $46.9 \%$ ( $>40 \%$ ) (i.e., resulting in $<60 \%$ baseline TER values; $p<0.001)$. Compared with the 


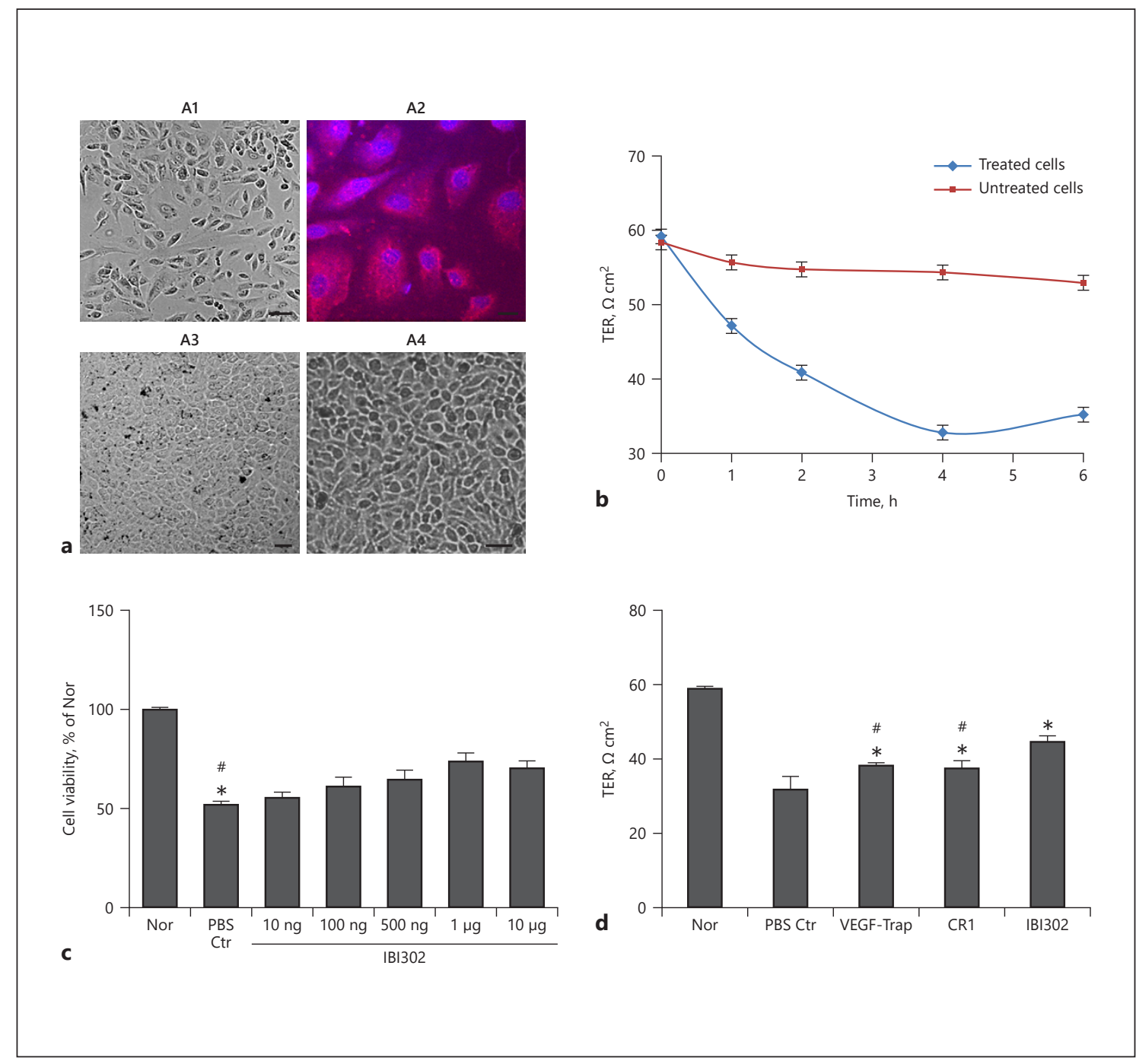

Fig. 1. a Identification of hRPE cells and the establishment of RPE monolayer barrier. P4 primary RPE cells were cultured $(\mathbf{A} 1, \times 200)$ and identified by RPE65 (A2, red, $\times 50)$. A stable RPE monolayer barrier was established about 21 days after cultured on transwell insert ( $\times 50($ A3); $\times 100($ A4)). b Oxidative stress in combination with NHS disrupted the function of RPE monolayer. After the hRPE cells reached about $80 \%$ confluence, $500 \mu \mathrm{M} \mathrm{t}-\mathrm{BHP}$ and $10 \%$ NHS were added to mimic the complement-activated oxidative stress in vitro. TER was recorded from 0 to $6 \mathrm{~h}$ after the treatment. Oxidative stress combined with complement activation significantly decreased the TER of RPE monolayer in a time-dependent manner ( $p<0.05$ vs. baseline), with a little rebound at $6 \mathrm{~h}$. No significant difference was found between 4 and $6 \mathrm{~h}(p=0.252)$. No significant difference existed between all the time points of TER in the untreated hRPE cells. The TER of untreated hRPE cells at 1, 2, 4 , and $6 \mathrm{~h}$ was significantly higher than that of the t-BHP and NHStreated hRPE cells. c IBI302 improved the cell viability of hRPE cells under the complement-activated oxidative stress. The optimal concentration of IBI302 was determined by MTT assay. Com- pared with the PBS control group, the different concentration of IBI302 improved the cell viability of hRPE cells under the complement-activated oxidative stress. No significant difference of cell viability was found between 1 and $10 \mu \mathrm{g} / \mathrm{mL}$ IBI302 group. $1 \mu \mathrm{g} /$ $\mathrm{mL}$ of IBI302 was chosen for the further experiments. d IBI302 restored the TER of RPE monolayer under the complement-activated oxidative stress. VEGF-Trap, CR1, and IBI302 reduced the TER reduction of RPE monolayer under $4 \mathrm{~h}$ of the complementactivated oxidative stress ( ${ }^{*} p<0.05$ vs. PBS group). IBI302 could restore the TER to $75.90 \%$ baseline TER value, which was significantly effective than VEGF-Trap $\left(64.64 \%,{ }^{*} p<0.05\right.$ vs. IBI302 group) and CR1 (63.48\%, ${ }^{*} p<0.001$ vs. IBI302 group). Nor, normal control, Ctr, PBS control. The experiments were repeated 3 times with 3 different batches of objects. Nor, normal control; Ctr, PBS control; TER, transepithelial resistance; VEGF, vascular endothelial growth factor; RPE, retinal pigment epithelial; hRPE, human RPE; t-BHP, ten-butyl hydroperoxide; NHS, normal human serum; CR1, complement receptor 1 . 


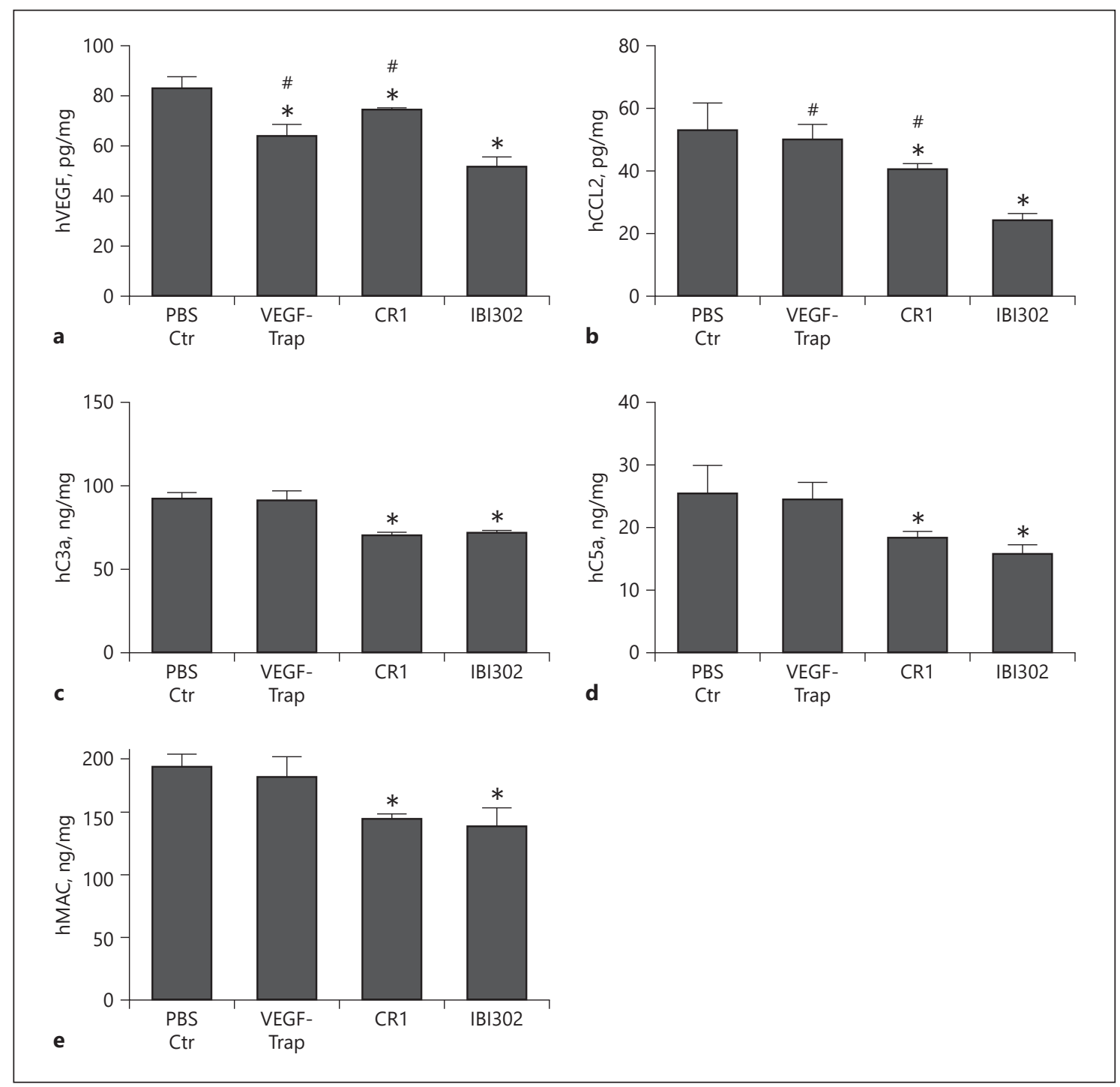

Fig. 2. IBI302 reduced the upregulation of VEGF, CCL2, and complement activation of hRPE under the complement-activated oxidative stress. IBI302 significantly reduced the increase of VEGF and CCL 2 compared with the VEGF-Trap and CR1 ( ${ }^{*} p<0.05$ vs. IBI302 group) and the PBS control group $\left({ }^{*} p<0.05\right.$ vs. PBS control group) (a, b). The levels of both VEGF and CCL2 in the IBI302 group were significantly lower than in the VEGF-Trap group and the CR1 group ( ${ }^{*} p<0.05$ vs. IBI302 group). IBI302 reduced the production of C3a, C5a, and MAC compared with $\mathrm{PBS}\left({ }^{*} p<0.05\right.$

PBS control group, VEGF-Trap, CR1, and IBI302 could significantly reduce the TER reduction of hRPE under the complement-activated oxidative stress $(p<0.05)$. IBI302 could restore the TER to $75.90 \%$ of the baseline TER values, which were significantly more effective than VEGF$\operatorname{Trap}(64.64 \%, p<0.05$ vs. IBI302 group) and CR1 (63.48\%, $p<0.001$ vs. IBI302 group). vs. PBS control group) (c-e). However, no significant difference existed between the IBI302 group and the CR1 group. Compared with PBS control group, VEGF-Trap treatment did not show difference at the levels of C3a, C5a, and MAC in hRPE cells. The experiments were repeated 3 times with 3 different batches of objects. CCL2, chemokine (C-C Motif) ligand 2; VEGF, vascular endothelial growth factor; CR1, complement receptor 1; Ctr, PBS control; hRPE, human retinal pigment epithelial; MAC, membrane attack complex.

\section{IBI302 Reduced the Increase of VEGF, C3a,}

C5a, MAC, and CCL2 in hRPE Cells under the

\section{Complement-Activated Oxidative Stress}

To determine the further effect of IBI302 on VEGF levels, complement activation and inflammatory response in hRPE cells under the complement-activated oxidative stress, the levels of VEGF, CCL2, and comple- 


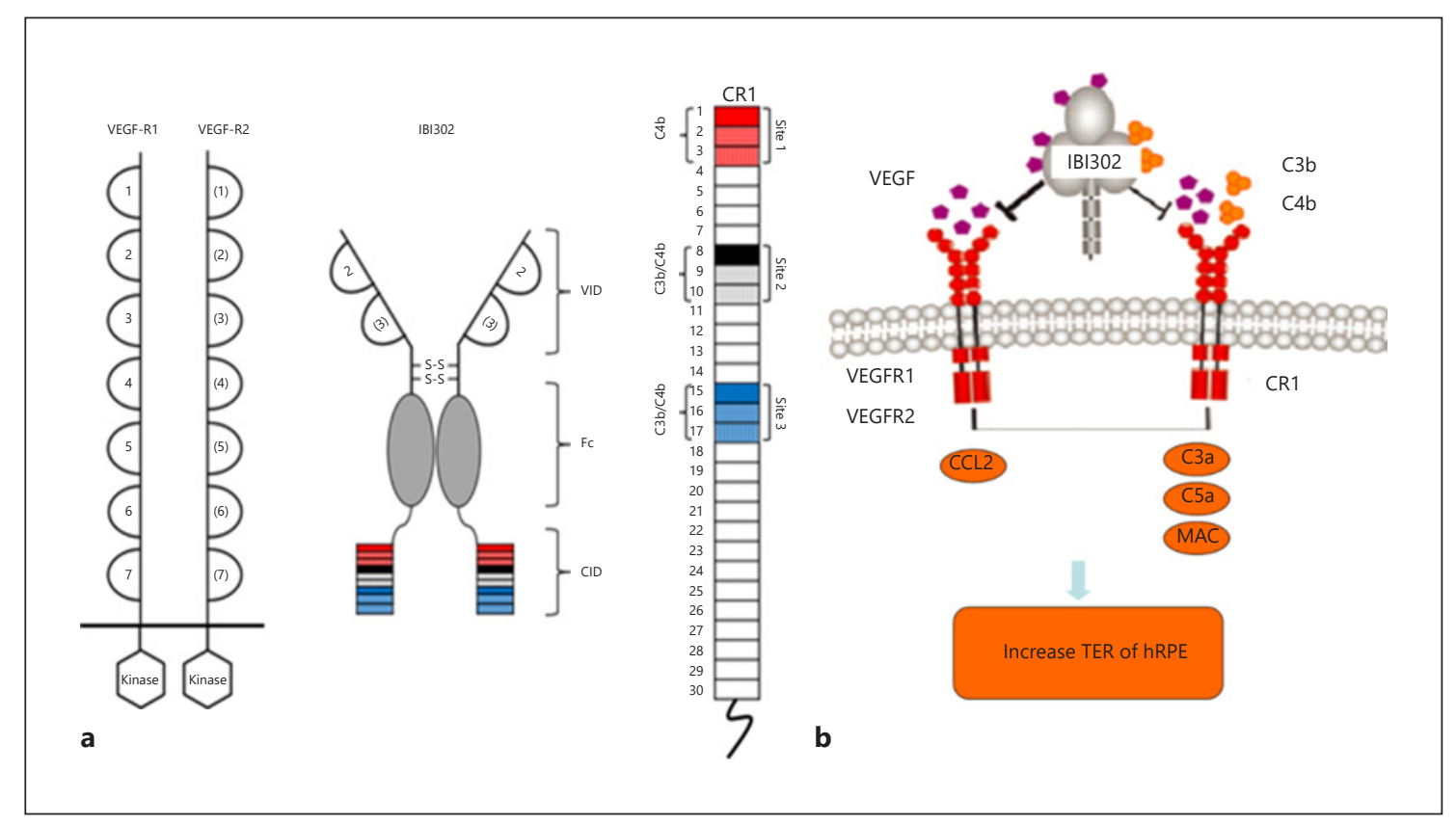

Fig. 3. a Molecular structure of IBI302. IBI302 is a recombinant VEGFR and CR1 fused to the Fc portion of human IgG1 with $100 \%$ human protein sequence. Its ligand-binding elements are taken from the extracellular domain 2 of VEGFR-1, the extracellular domain 3 of VEGFR-2 as well as the C4b and C3b/C4b-binding por-

ment activation products $\mathrm{C} 3 \mathrm{a}, \mathrm{C} 5 \mathrm{a}$, and $\mathrm{MAC}$ were measured by ELISA (shown in Fig. 2). IBI302 significantly reduced the levels of VEGF and CCL2 by 59.53 and $54.21 \%$ (from an average value of 83.3 to $49.6 \mathrm{pg} / \mathrm{mg}$ and 53 to $29.0 \mathrm{pg} / \mathrm{mg}$, respectively) compared with the PBS control group $(p<0.05)$ (shown in Fig. 2a, b). The levels of both VEGF and CCL2 in the IBI302-treated group were significantly lower than that in the VEGF-Trap and the CR1-treated groups ( $p<0.05$ vs. IBI302 group). IBI302 also significantly reduced the production of C3a, C5a, and MAC than PBS $(p<0.05)$ (shown in Fig. $2 c-e)$. However, there was no significant difference in the levels of C3a, C5a, and MAC between the IBI302 and the CR1treated groups or between the VEGF-Trap-treated group and the PBS control group.

\section{Discussion}

As IBI302 is a bispecific fusion protein with the ligands taken from VEGFR1, VEGFR2, and CR1 (shown in Fig. 3a), we first detected the combination effect of IBI302 on the VEGF and immune response products such as $\mathrm{C} 3 \mathrm{a}, \mathrm{C} 5 \mathrm{a}$, and $\mathrm{MAC}$ and investigated its protective effect tions of CR1. b The mechanism of action of IBI302. VEGF, vascular endothelial growth factor; VEGFR, VEGF receptor; hVEGF, human VEGF; CR1, complement receptor 1; TER, transepithelial resistance; hRPE, human retinal pigment epithelial.

on the barrier of hRPE in vitro. To mimic the oxidative stress and complement activation of hRPE in AMD, 500 $\mu \mathrm{M} \mathrm{t}$-BHP was used to introduce oxidative stress to hRPE monolayer barrier, and 10\% NHS was used to provide complement components [22]. Our study found that IBI302 could downregulate VEGF more intensively than CR1 and VEGF-Trap and also decreased the expression of C3a, C5a, and MAC as CR, while VEGF-Trap showed no effect on the complement system.

MAC is formed from the binding of $\mathrm{C} 5 \mathrm{~b}$ to the plasma complement proteins, which leads to cell lysis and death, or changes in cellular responses such as secretion, adherence, aggregation, or cell division [25-27]. C3a and C5a, the bioactive fragments of C3 and C5 during complement activation, have been reported to exist in the drusen of patients with AMD [28]. VEGF is one of the main growth factors which can disrupt the barrier function of RPE cells [29-31], while MAC, C3a, and C5a can induce the increasing of VEGF and promote the disruption of the barrier [24]. It has already been reported that MAC, C3a, and C5a can induce the increasing of VEGF both in vitro and in vivo [24, 32]. In view of an attenuated VEGF expression by CR1 but no significant expression by VEGF-Trap, we could infer that VEGF 
might be on the downstream of the complement activation pathway as hypothesized in another research [33]. This study indicated that IBI302 treatment significantly reduced complement activation by $\mathrm{CR} 1$ targeting $\mathrm{C} 3 \mathrm{~b}$ and $\mathrm{C} 4 \mathrm{~b}$, then further downregulated the levels of $\mathrm{C} 3 \mathrm{a}$, $\mathrm{C} 5 \mathrm{a}$, and MAC, and then contributed an indirect attenuated VEGF level.

Moreover, we analyzed the level of hCCL-2 in the supernatant of hRPE with ELISA; IBI302 showed greater reduction on hCCL-2 than CR1, while VEGF-Trap had no effect on hCCL-2. CCL2 level is very low in normal RPE but increases in acute inflammation with aging or when the RPE cells are under oxidative stress $[34,35]$. The inflammatory cytokine CCL-2 plays an important role during the process of AMD [36]. MAC modulates the production and secretion of CCL2 and then further increases VEGF level [24]. Our study found that IBI302 reduced the level of inflammatory cytokine CCL2, as well as the complement activation products such as $\mathrm{C} 3 \mathrm{a}, \mathrm{C} 5 \mathrm{a}$, and MAC. The underlying mechanism of IBI302 upon the complement response might lie in the $\mathrm{C} 3 \mathrm{a}, \mathrm{C} 5 \mathrm{a}$, and MAC-mediated downregulation of CCL-2, followed by VEGF decreasing. The mechanism of IBI302 on the barrier of cultured hRPE cells under the complement-activated oxidative stress is shown on the schematic plot (shown in Fig. 3b).

In conclusion, this study suggested that IBI302 could protect the barrier function of RPE cells against the complement-activated oxidative stress by downregulating the $\mathrm{C} 3 \mathrm{a}, \mathrm{C} 5 \mathrm{a}$, and MAC-mediated inflammatory response and the levels of VEGF. IBI302 exhibited the protective effect for the barrier function of hRPE cells and might be a potentially effective drug for the RPE barrier-associated ocular diseases.

\section{Acknowledgement}

This study does not include any nonauthor contributors to acknowledge.

\section{Statement of Ethics}

Donors were fully aware that donated eyes are used for treatment or research purposes, and written consent for eye donation was obtained from all donors. This study was conducted in accordance with the Declaration of Helsinki and approved by the Medical Ethics Committee of Zhongshan Ophthalmic Center, Sun Yatsen University.

\section{Conflict of Interest Statement}

The authors of this study do not have any conflict of interest to declare.

\section{Funding Sources}

This work was supported by the grants from the National Natural Science Foundation of China to Y. L. (81371020).

\section{Author Contributions}

Jing Wang completed cell experiments and wrote the paper. Yishen Wang wrote the paper. Qiuhui Liu, Shaofen Lin, and Rong Tian supported the cell culture. Dechao Yu and Jia Li supplied the IBI302. Dechao Yu reviewed the paper and gave suggestions on it. Yan Luo contributed to the experiment design and paper supervision.

\section{References}

1 Du H, Xiao X, Stiles T, Douglas C, Ho D, Shaw PX. Novel mechanistic interplay between products of oxidative stress and components of the complement system in AMD pathogenesis. Open J Ophthalmol. 2016;6(1):43-50.

2 Khan JC, Shahid H, Thurlby DA, Bradley M, Clayton DG, Moore AT, et al. Age related macular degeneration and sun exposure, iris colour, and skin sensitivity to sunlight. $\mathrm{Br} J$ Ophthalmol. 2006;90(1):29-32.

3 Mitchell P, Liew G, Gopinath B, Wong TY. Age-related macular degeneration. Lancet. 2018;392(10153):1147-59.

4 Dong A, Xie B, Shen J, Yoshida T, Yokoi K, Hackett SF, et al. Oxidative stress promotes ocular neovascularization. J Cell Physiol. 2009;219(3):544-52.
5 Lai K, Xu L, Jin C, Wu K, Tian Z, Huang C, et al. Technetium-99 conjugated with methylene diphosphonate (99Tc-MDP) inhibits experimental choroidal neovascularization in vivo and VEGF-induced cell migration and tube formation in vitro. Invest Ophthalmol Vis Sci. 2011;52(8):5702-12.

6 Holz FG, Schmitz-Valckenberg S, Fleckenstein M. Recent developments in the treatment of age-related macular degeneration. J Clin Invest. 2014;124(4):1430-8.

7 Kaarniranta K, Sinha D, Blasiak J, Kauppinen A, Veréb Z, Salminen A, et al. Autophagy and heterophagy dysregulation leads to retinal pigment epithelium dysfunction and development of age-related macular degeneration. Autophagy. 2013;9(7):973-84.
8 Tan LX, Toops KA, Lakkaraju A. Protective responses to sublytic complement in the retinal pigment epithelium. Proc Natl Acad Sci U S A. 2016;113(31):8789-94.

9 Bora NS, Matta B, Lyzogubov VV, Bora PS Relationship between the complement system, risk factors and prediction models in age-related macular degeneration. Mol Immunol. 2015;63(2):176-83.

10 Gondwe EN, Molyneux ME, Goodall M, Graham SM, Mastroeni P, Drayson MT, et al. Importance of antibody and complement for oxidative burst and killing of invasive nontyphoidal Salmonella by blood cells in Africans. Proc Natl Acad Sci U S A. 2010;107(7):30705.

Wang/Wang/Yu/Liu/Lin/Tian/Li/Luo 
11 Lachmann PJ. Preparing serum for functional complement assays. J Immunol Methods. 2010;352(1-2):195-7.

12 Yip PP, Woo CF, Tang HH, Ho CK. Triple therapy for neovascular age-related macular degeneration using single-session photodynamic therapy combined with intravitreal bevacizumab and triamcinolone. $\mathrm{Br} \mathrm{J} \mathrm{Oph}$ thalmol. 2009;93(6):754-8.

13 Hollborn M, Ackmann C, Kuhrt H, Doktor F, Kohen L, Wiedemann P, et al. Osmotic and hypoxic induction of the complement factor C9 in cultured human retinal pigment epithelial cells: regulation of VEGF and NLRP3 expression. Mol Vis. 2018;24:518-35.

14 Bora PS, Sohn JH, Cruz JM, Jha P, Nishihori $\mathrm{H}$, Wang $\mathrm{Y}$, et al. Role of complement and complement membrane attack complex in laser-induced choroidal neovascularization. J Immunol. 2005;174(1):491-7.

15 Ao J, Wood JP, Chidlow G, Gillies MC, Casson RJ. Retinal pigment epithelium in the pathogenesis of age-related macular degeneration and photobiomodulation as a potential therapy? Clin Exp Ophthalmol. 2018; 46(6):670-86

16 Khandhadia S, Cipriani V, Yates JR, Lotery AJ. Age-related macular degeneration and the complement system. Immunobiology. 2012; 217(2):127-46.

17 Ren X, Li J, Xu X, Wang C, Cheng Y. IBI302, a promising candidate for AMD treatment, targeting both the VEGF and complement system with high binding affinity in vitro and effective targeting of the ocular tissue in healthy rhesus monkeys. Exp Eye Res. 2016; 145:352-8.

18 Augustin AJ, Offermann I. Combination therapy for choroidal neovascularisation. Drugs Aging. 2007;24(12):979-90.

19 Ablonczy Z, Crosson CE. VEGF modulation of retinal pigment epithelium resistance. Exp Eye Res. 2007;85(6):762-71.
20 Rabin DM, Rabin RL, Blenkinsop TA, Temple $\mathrm{S}$, Stern JH. Chronic oxidative stress upregulates Drusen-related protein expression in adult human RPE stem cell-derived RPE cells: a novel culture model for dry AMD. Aging. 2013;5(1):51-66.

21 Hanus J, Zhang H, Chen DH, Zhou Q, Jin P, Liu Q, et al. Gossypol acetic acid prevents oxidative stress-induced retinal pigment epithelial necrosis by regulating the FoxO3/sestrin2 pathway. Mol Cell Biol. 2015;35(11):1952.

22 Thurman JM, Renner B, Kunchithapautham K, Ferreira VP, Pangburn MK, Ablonczy Z, et al. Oxidative stress renders retinal pigment epithelial cells susceptible to complementmediated injury. J Biol Chem. 2009;284(25): 16939-47.

23 Jungnickel J, Claus P, Gransalke K, Timmer M, Grothe C. Targeted disruption of the FGF2 gene affects the response to peripheral nerve injury. Mol Cell Neurosci. 2004;25(3):44452.

24 Liu J, Jha P, Lyzogubov VV, Tytarenko RG, Bora NS, Bora PS. Relationship between complement membrane attack complex, chemokine (C-C motif) ligand 2 (CCL2) and vascular endothelial growth factor in mouse model of laser-induced choroidal neovascularization. J Biol Chem. 2011;286(23):20991-1001.

25 Bohana-Kashtan O, Ziporen L, Donin N, Kraus S, Fishelson Z. Cell signals transduced by complement. Mol Immunol. 2004;41(67):583-97.

26 Chen Y, Yang C, Jin N, Xie Z, Tang Y, Fei L, et al. Terminal complement complex C5b9-treated human monocyte-derived dendritic cells undergo maturation and induce Th1 polarization. Eur J Immunol. 2007;37(1):16776.

27 Xie CB, Jane-Wit D, Pober JS. Complement membrane attack complex: new roles, mechanisms of action, and therapeutic targets. Am J Pathol. 2020;190(6):1138-50.
28 Hageman GS, Luthert PJ, Victor Chong NH, Johnson LV, Anderson DH, Mullins RF. An integrated hypothesis that considers drusen as biomarkers of immune-mediated processes at the RPE-Bruch's membrane interface in aging and age-related macular degeneration. Prog Retin Eye Res. 2001;20(6):705-32.

29 Hartnett ME, Lappas A, Darland D, McColm JR, Lovejoy S, D'Amore PA. Retinal pigment epithelium and endothelial cell interaction causes retinal pigment epithelial barrier dysfunction via a soluble VEGF-dependent mechanism. Exp Eye Res. 2003;77(5):593-9.

30 Ghassemifar R, Lai CM, Rakoczy PE. VEGF differentially regulates transcription and translation of ZO-1alpha+ and ZO-1alphaand mediates trans-epithelial resistance in cultured endothelial and epithelial cells. Cell Tissue Res. 2006;323(1):117-25.

31 Bird AC. Therapeutic targets in age-related macular disease. J Clin Invest. 2010;120(9): 3033-41

32 Nozaki M, Raisler BJ, Sakurai E, Sarma JV, Barnum SR, Lambris JD, et al. Drusen complement components $\mathrm{C} 3 \mathrm{a}$ and $\mathrm{C} 5 \mathrm{a}$ promote choroidal neovascularization. Proc Natl Acad Sci U S A. 2006;103(7):2328-33.

33 Girardi G, Yarilin D, Thurman JM, Holers VM, Salmon JE. Complement activation induces dysregulation of angiogenic factors and causes fetal rejection and growth restriction. J Exp Med. 2006;203(9):2165-75.

34 Chen H, Liu B, Lukas TJ, Neufeld AH. The aged retinal pigment epithelium/choroid: a potential substratum for the pathogenesis of age-related macular degeneration. PLoS One. 2008;3(6):e2339.

35 Chen Y, Zhong M, Yuan G, Peng H. Interleukin-17 induces angiogenesis in vitro via CXCL8 and CCL2 in retinal pigment epithelium. Mol Med Rep. 2018;17(3):4627-32.

36 Sharma NK, Sharma K, Singh R, Sharma SK, Anand A. CCL2 single nucleotide polymorphism of rs 1024611 implicates prominence of inflammatory cascade by univariate modeling in Indian AMD. PLoS One. 2018;13(4): e0193423. 